\title{
3D image modelling and specific treatments in orthodontics domain
}

doi:10.1080/11762320701754753

\author{
Dionysis Goularas ${ }^{1}$, Khalifa Djemal ${ }^{2}$ \\ and Yannis Mannoussakis ${ }^{1}$ \\ ${ }^{1}$ L.R.I Laboratoire de Recherche en Informatique, Bât 490 Université Paris-Sud, 91405 Orsay, France \\ ${ }^{2}$ I.B.I.S.C Laboratoire d'Informatique, Biologie Intégrative et Systèmes Complexes, \\ 40 rue du Pelvoux, 91020 Evry, France
}

\begin{abstract}
In this article, we present a 3D specific dental plaster treatment system for orthodontics. From computer tomography scanner images, we propose first a 3D image modelling and reconstruction method of the Mandible and Maxillary based on an adaptive triangulation allowing management of contours meant for the complex topologies. Secondly, we present two specific treatment methods directly achieved on obtained 3D model allowing the automatic correction for the setting in occlusion of the Mandible and the Maxillary, and the teeth segmentation allowing more specific dental examinations. Finally, these specific treatments are presented via a client/server application with the aim of allowing a telediagnosis and treatment.
\end{abstract}

Key words: 3D specific dental plaster treatment, 3D reconstruction, teeth segmentation, topology changes management, orthodontics domain

\section{INTRODUCTION}

For some years now, the research works in image processing and 3D visualization systems applied to medical images aim at the conception and implementation of diagnosis help systems, in order to treat and analyse specific examinations. The $3 \mathrm{D}$ reconstruction allows a global perception, a localization of lesions in regions that are not targeted by doctors, or the information storage and transmission. The complexity of the treated images makes the automatic interpretation difficult, because the treatment must satisfy the material environment constraints and also those of the specialists. In spite of these problems, an important development of tools and computer methods is being achieved in the medicine domain, aiming to store and process medical images. Presently, many tools exist that help digitize, treat and exchange patient's examinations. Despite this development some domains, such as

Corresponding Author:

Dr Khalifa Djemal

I.B.I.S.C Laboratoire d'Informatique

Biologie Intégrative et Systèmes Complexes

40 rue du Pelvoux

91020 Evry

France

Tel: 0033-169-477-554

Email: djemal@iup.univ-evry.fr orthodontics, still remain where doctors cannot avail the advantage offered by computer technology. Indeed, the majority of orthodontists continue to work essentially with traditional methods focused on the physical dental plaster such as teeth measures, physical storage of dental plasters, physical cut of the plaster teeth, taking photos, etc. In particular, the numeric dental plaster treatment and storage attract our attention because of the big number of the subjects that must be treated, 30-50 cases by month for a middle laboratory. In this article, we propose a $3 \mathrm{D}$ modelling and specific treatment system for dental plasters via a client/server application in order to contribute to the efforts for faster and better diagnosis in orthodontics domain. To develop the orthodontics system, three stages are necessary, 3D modelling and reconstruction, orthodontics specific treatments and client/server application allowing the telediagnosis.

In general, the different methods of 3D image reconstruction are based on two approaches: firstly, the structured methods by the models that they use (ter Haar 1999), but they are limited in their diversity by these same models. The second methods are little structured by the lowlevel or primitive models that they use (Fetita and Preteux 2000); therefore, the data structuring takes place after the reconstruction step. Orthodontics follows this effort, and the need to treat the digitalized data of patient increases constantly (Motohashi et al. 1999; Marcel 2001; Redmond 2001). 3D modelling and reconstruction in this domain 


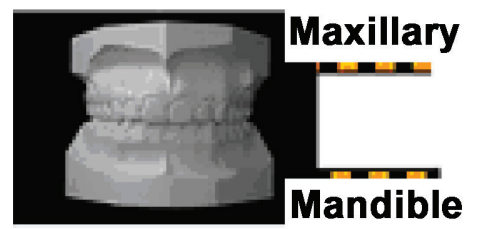

a

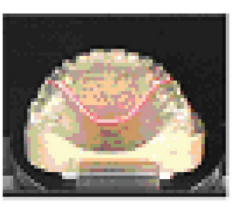

b

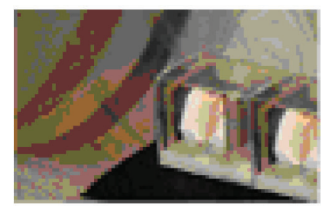

c

Figure 1 (a) Dental plaster, (b) surface scanner and (c) CT scanner.

are topics that are little exploited and remain among those many treatment problems that have inadequate systems allowing the exploitation and image processing. In this domain, the acquirement methods use mainly 3D surface scanners (Figure 1.b), with laser beam (Redmond 2001). The result of this method is a cloud of points of the scanned object. The triangulated surface is given from a supplementary procedure based on this same cloud of points. The main advantages of this method are the affordable price of the surface scanners and the high resolution of the obtained models. The generation of the triangulated surface model and the setting in occlusion of the two 3D sub-models (Maxillary, Mandible) constitute its main disadvantage since they considerably increase the creation time of digital plasters.

In the proposed work, the dental plaster images are obtained by CT (computed tomography) scanner (Figure 1.c). This system presents several advantages. First, we can digitalize a dental plaster model more quickly. Then we have the possibility to digitalize several successive dental plasters in an entirely automatic manner. In our approach, the 3D modelling method is based on a set of contours obtained from each segmented image of the CT scanner sequence. These contours are the result of the segmentation of objects that are present in some or each layer of the 3D images. This result follows a hierarchical structure (Figure 2). This structuring is the base for the development of procedures necessary to our 3D reconstruction algorithm. We apply this process on the two sequences of images that correspond to the Maxillary and the Mandible. The two obtained sub-models are adequately combined to achieve the global $3 \mathrm{D}$ model of the dental plaster. After the creation of the 3D dental plaster model, the specific treatments according to the orthodontists' needs require the appropriate methods that we

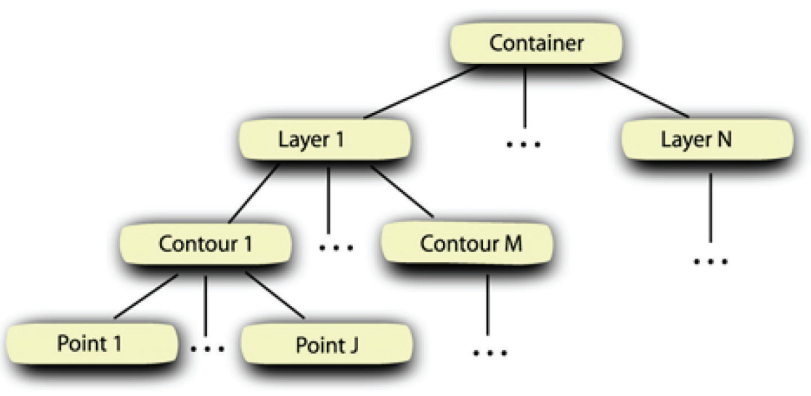

Figure 2 Segmented data structuring. propose: teeth measures and an automatic correction of the dental occlusion, done directly on the obtained 3D model.

To try satisfying the orthodontics needs, these methods are presented via a client/server application specialized in this domain. This application allows the visualization and the digital examination treatment with special telediagnosis tools. The main advantages of this application are the fast creation of digital examinations, the data management and the diagnosis tools, giving the possibility to orthodontists to have an efficient digital solution for the storage and treatment of their examinations.

This article is organized in the following way: in section 2 , we present a $3 \mathrm{D}$ reconstruction method by adaptive triangulation intended to complex structures. This method allows generating a surface between two closed contours contained in two successive layers and also manages the changes of topologies in images' sequence. The specific $3 \mathrm{D}$ treatments are presented in section 3 . We first present an automatic correction method for the setting in occlusion of the two sub-models, achieved directly on the obtained 3D model and, secondly, a semiautomatic segmentation method allowing an individual treatment of teeth. In section 4, we present the DIVITOR (DIgitization, Visualisation and Treatment in ORthodontics) client/server application. The specific treatments and the obtained results are showed via the DIVITOR application in section 5.

\section{D IMAGE MODELLING BY ADAPTIVE TRIANGULATION METHOD WITH COMPLEX TOPOLOGY MANAGEMENT}

The topology changes' complexity in image processing aroused the curiosity among a great number of researchers. Indeed, this problem relates to the organic structure in medical images, such as the arterial and venous structures or generally the structures of complex topology like dental structure. Among the existing reconstruction methods, the Marching Cubes algorithm (Lorensen and Cline 1987) can carry out a $3 \mathrm{D}$ reconstruction managing successfully the complex topology of these structures but requires a great memory to be able to treat the obtained models. In fact, with the Marching Cubes algorithm, in the case of dental plasters, the air that is trapped within the plasters creates a significant number of surfaces that are not necessary for the dental examinations. These surfaces are considerably augmented in the case of dental prints. To obtain a very consistent and accurate model, many $3 \mathrm{D}$ reconstruction 
methods are developed. These methods are based on contours allowing a better visualization and treatment in different domains. In this topic we distinguish two complexity levels for the management of the topology changes. In the first level, in a 2D field, several separation methods were developed to deal with the management of topology. The majority of these methods are based on deformable models where the changes of topology are managed implicitly using the level set formulation (Malladi et al. 1995; Djemal et al. 2006). Others tried to solve this problem by the definition of topological operators adapted to the studied structure (Rey et al. 1999), but it is difficult to generalize them; therefore, results can be obtained only on synthetic models. The second level of topological changes' management is related with the $3 \mathrm{D}$ reconstruction of the model.

Indeed, there are authors who deal with the problem of the 3D reconstruction starting from contours (Oliva et al. 1996; Klein et al. 2000). However, these methods were often intended for the reconstruction of relatively simple structures. Indeed, these methods are limited by several constraints and are based on prior information of the studied shape (Lee et al. 1999). It is within this framework that the method we present is registered.

\section{Adaptive triangulation method}

In the domain of structures' reconstruction, many triangulation methods have been developed and proposed in literature. An adaptive triangulation refinement method is presented in Persiano et al. (1993). In this work, the authors consider the refinement as minimal in the number of elements (triangles). This method presents acceptable results on simple structures, but in the case of the complex structures, the topology changes are not taken into account. In the same way, Stämpfle et al. (1999) use an adaptive triangulation method for the numerical computation of flow approximations. The limit of this method as evaluated by the authors concerns the refinements that involve integration costs for each new vertex. Furthermore, topology changes are not considered in their $3 \mathrm{D}$ reconstruction system. A 3D reconstruction of complex shapes based on the Delaunay triangulation is presented in (Boissonnat et al. 1992). In their approach, they use a Voronoi skeleton where the end cap surfaces are triangulated. This method needs a lot of computation time, and the results depend on prior information of complex shapes.

A triangulation algorithm is proposed by Scheidegger et al. (2005). This triangulation is obtained directly from point set surfaces. The refinement is based on moving least squares surfaces, but there are very few theoretical results on moving least squares surfaces that can be used in practice. In this work, the topology changes' complexity is not introduced.

The adaptive triangulation method that we present is based on geometrical and topological criteria. This method aims to generate a $3 \mathrm{D}$ object starting from two sets of

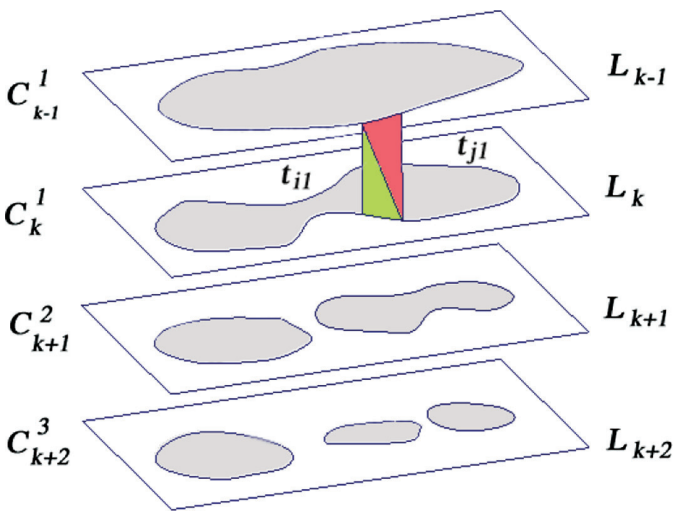

Figure 3 Example of topology change on successive images.

ordered points describing two contours contained in two successive images (layers).

Let a container be defined like a set of layers where each laver corresponds to the contents of a 2D image. Each layer is defined as a whole of contours where each contour contains a whole of points. Figure 2 illustrates the structure of data obtained by the 2D images where 2D contours are calculated. Our approach is based on the segmentation of 2D images data using a deformable active contour model (Djemal et al. 2002). This method allows obtaining closed contours and also manages the 2D topology changes.

Let, a contour $C_{k}^{m}$ belonging to an image $L_{k}$ is regarded as a series of the successive points $P_{k}$ with $L_{k} \in$ $\left\{L_{1}, \ldots, L_{l}\right\}$ and $m$ the number of contours in layer $L_{k}$. For example, layer $k+1$ (Figure 3 ) contains two contours $(m=2)$. These contours can be noted $\Gamma_{1}$ and $\Gamma_{2}$, the set of points of the two contours in layer $k+1$ is considered as following:

$P_{k+1}=\left\{\Gamma_{1}, \Gamma_{2}\right\}$ with $\Gamma_{1}=\left\{q_{1}, \ldots, q_{\theta}\right\}$ and $\Gamma_{2}=$ $\left\{q_{\theta+1}, \ldots, q_{N}\right\}$.

The global set of points describing all contours $C_{k+1}^{2}$ in this layer is $P_{k+1}=\left\{q_{1}, \ldots, q_{\theta}, q_{\theta+1}, \ldots, q_{N}\right\}$.

If we consider that the contours $C_{k}^{1}$ and $C_{k-1}^{1}$ are contained in the images $L_{k}$ and $L_{k-1}$ (Figure 3), we have the two sets of points $P_{k}$ and $P_{k-1}$ describing these two contours:

$$
P_{k-1}=\left\{p_{1}, \ldots, p_{N}\right\} \quad \text { and } \quad P_{k}=\left\{q_{1}, \ldots, q_{M}\right\} .
$$

We want to define the totality of the triangles that constitute the closed surface $S$ between two contours $C_{k}^{1}$ and $C_{k-1}^{1}$.

A surface between two contours can be approximated by various geometrical forms controlled by a mathematical equation. We chose the triangle for two reasons. First, a triangle allows describing a surface with just three points. Secondly, the graphics boards use the triangles for the surface representation. The creation of surface $S$ is carried out in two stages, first the localization of the point $q_{j}$ in $P_{k}$ nearest to each point $p_{i}$ of the totality of points in $P_{k-1}$ by using a neighbourhood criterion. This enables to 
generate the set $T_{k-1}$ of triangles $t_{i 1}$. The second stage consists in testing the totality of points in $P_{k}$ of $C_{k}^{1}$ by seeking the missing triangles $t_{j}$ of the set $T_{k}$ by considering the membership of the points $q_{j}$ of $P_{k-1}$ at the triangles $t_{i 1}$ already created with the previous stage.

$$
T_{k-1}=\sum_{i 1=1}^{T N} t_{i 1}, \quad T_{k}=\sum_{j 1=1}^{T M} t_{j 1},
$$

with $T N$ the triangles number of $T_{k-1}$ and $T M$ the triangles number of $T_{k}$. So we obtain a surface $S$ by linking the two sets $T_{k-1}$ and $T_{k}$ :

$$
S=T_{k-1} \cup T_{k}=\sum_{i 1=1}^{T N} t_{i 1}+\sum_{j 1=1}^{T M} t_{j 1}
$$

\section{Generation of the triangles $T_{k-1}$ and $T_{k}$}

For each point $p_{i} \in P_{k-1}$, we seek the nearest point $q_{j} \in$ $P_{k}$ using the corresponding Euclidean distance $d_{i}$. The distance $d_{i}$ is implicitly calculated to find the optimal point $q_{j}$; this point is used as a reference to find all the remainder of the neighbour points to $p_{i}$. This process enables to define a correspondence table of neighbourhood $V\left(P_{k-1}, P_{k v}\right)$, where $P_{k v}$ is the obtained corresponding points in contour $C_{k}^{1}$. The minimal criterion of distance can introduce errors into certain regions with strong considered curvature of contour $C_{k}^{1}$. Indeed, the points $p$ of contour $C_{k-1}^{1}$ can have several common points $q$ of correspondences belonging to $C_{k}^{1}$. These strong curvatures can thus cause discontinuities between the triangles because certain points $q$ cannot be taken into account. To solve this problem, we correct the set of the points $P_{k v}$ of the neighbour correspondences based on the continuity and curvature of the set of the points $P_{k}$ of $C_{k}^{1}$. We obtain, then, the corrected set of points $P_{k c}$ and the new correspondence corrected table $V_{c}\left(P_{k-1}, P_{k c}\right)$.

The creation of triangles $T_{k}$ is based on the contained information in the triangles of $T_{k-1}$. Indeed, in the first step, we are basing on the points of contour $C_{k}^{1}$ and for each point $q_{j} \in C_{k}^{1}$, which was selected like neighbour point to $C_{k-1}^{1}$, we generate a triangle $t_{j} \in T_{k}$ according to its participation in the formation of the triangle $t_{i} \in T_{k-1}$. For all the points of $C_{k}^{1}$, which do not belong to the set of the points $P_{k c}$, we create the triangles according to triangle

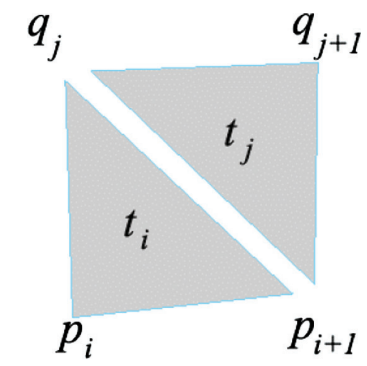

Figure 4 Triangles belonging to $T_{k-1}\left(t_{i}\right)$ and $T_{k}\left(t_{j}\right)$.

membership information of the points in the set $P_{k c}$. Figure 4 illustrates the components of the triangles belonging to $T_{k-1}$ and $T_{k}$, and the names of the points of these triangles that we allotted.

According to the attribution of a neighbour at the points $q \in C_{k}^{1}$, three cases can be distinguished. In case $1, q_{j} \in P_{k c}$, then $q_{j}$ is a top of triangle $t_{i}\left(p_{i}, p_{i+1}, q_{j}\right)$. In this first case (Figure 5.a), we carry out the creation of a triangle $t_{j}$, and we adapt it with the set $T_{k}$ such as $t_{j}\left(q_{j}, q_{j+1}, p_{i+1}\right)$. If the point $q_{i+1}$ does not belong to $P_{k c}$, the triangle $t_{j}$ is associated to it $q_{i+1} \in t_{j}$. In the second case, where $q_{j} \notin P_{k c}$, but its neighbourhood $q_{i-1} \in t_{j-1}$, the point $q_{i}$ does not belong to neighbourhood of points of $C_{k-1}^{1}$, but the point that precedes it is associated to triangle $t_{j-1}$ (Figure 5.b and Figure 5.c). The triangle that we generate is $t_{j}\left(q_{j}, q_{j+1}, p_{i+1}\right)$ where $p_{i+1} \in t_{j-1}$, and we can associate triangle $t_{j}$ to $q_{j}$ and $q_{j+1}$.

The last case concerns $q_{j} \notin P_{k c}$ and its neighbour $q_{j-1}$ is not a part of the tops of the triangle $t_{j-1}$. The point $q_{j}$ does not belong to neighbour of points of $C_{k-1}^{1}$ and in more the point $q_{j-1}$ does not belong to a triangle (Figure 5.d). This case occurs if, at the beginning of the procedure of the generation of $T_{k}, q_{i}$ and $q_{i-1} \notin P_{k c}$. We seek the nearest point $q_{j+u}$ which belongs to $P_{k c}$. As in the previous case, we associate $t_{j}$ to $q_{j}$ and $q_{j+1}$.

Figure 6 presents the adaptive triangulation method where the described cases are considered.

\section{Topology changes management method}

According to the shape geometry of which we want to carry out the $3 \mathrm{D}$ reconstruction, it is possible that between two consecutive images (layers) there are several contours to be associated. For example, between two images, we can

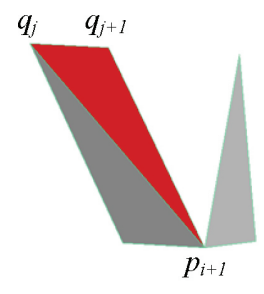

$\boldsymbol{a}$

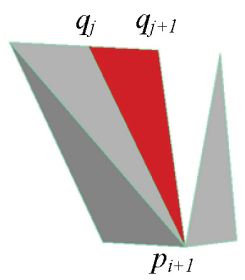

b

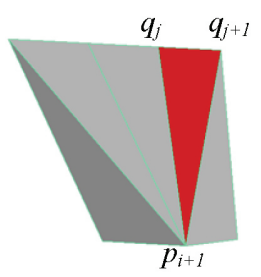

$c$

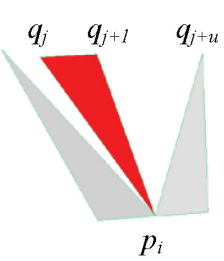

d

Figure 5 (a) case 1: $q_{i}$ belongs to the whole of the neighbour $P_{k c}$. (b and c) case 2: $q_{j}$ belonging to $T_{k}$; it is then associated to triangle $t_{j-1}$. (d) case $3: q_{j}$ is isolated. We seek the first neighbour $q_{j+u}$ to find the point $p_{i}$. 


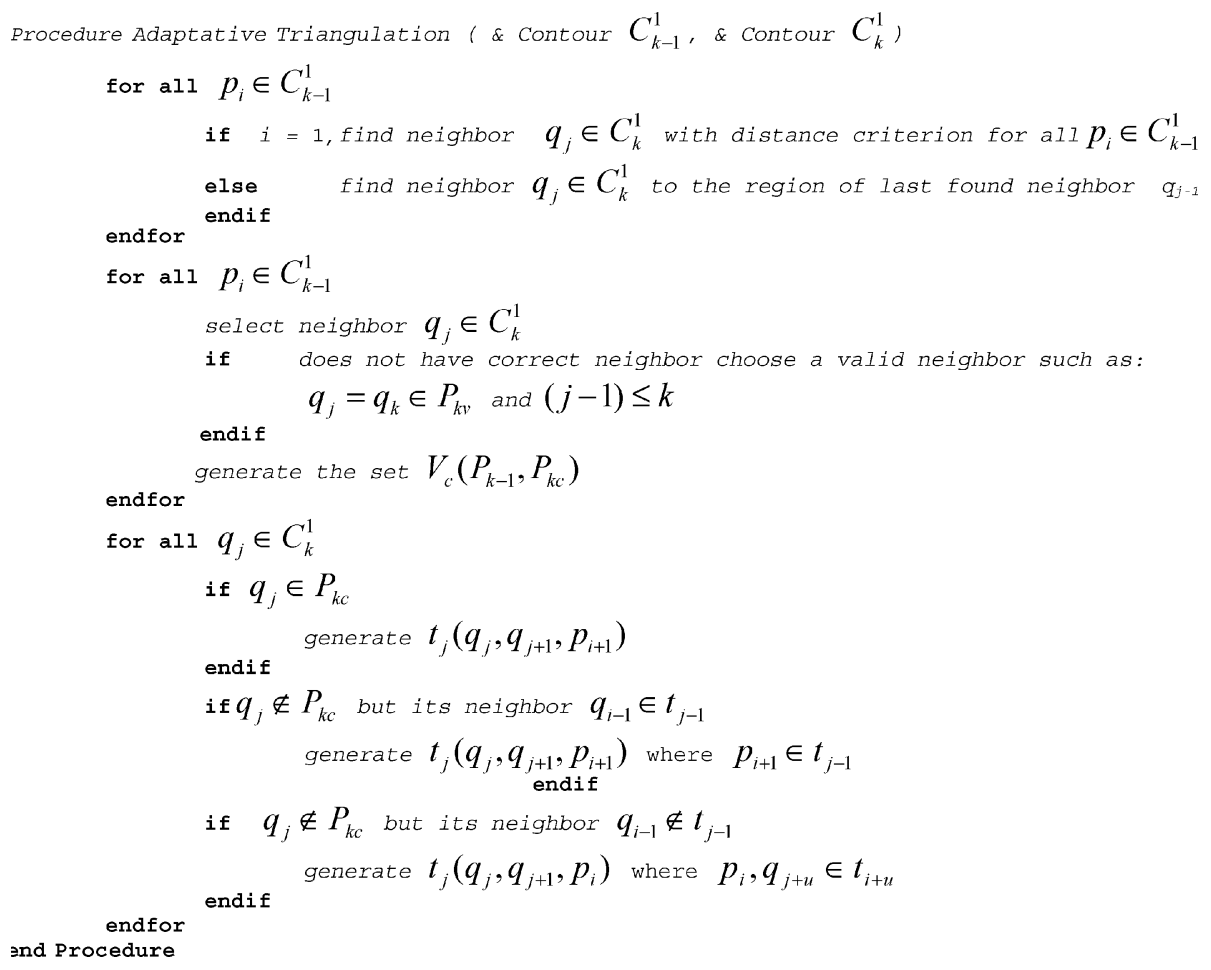

Figure 6 Adaptive triangulation algorithm.

have correspondences without a sub-division of a contour, a topology with a simple division (a contour is splitting in two contours), a topology with a complex division (a contour is splitting in many contours), or contours which do not have a correspondence between two images. The definition of regions' correspondence is an open research problem if the number of contours of an object varies between consecutive $2 \mathrm{D}$ images. The difficulties result from the great diversity of the topological cases according to the shape of the object and their possible solutions. We interest in this section to the definition of contour regions' separation considering that we treat complex shapes with a possible change of topology.

Firstly, we consider the case of topology with simple division. In this case, we are interested to the neighbourhood regions of two contours, and we seek in the preceding contour ('father' contour) the closest points belonging to the region defined before. These points will be the points of reference for the sub-division of the 'father' contour. Secondly, we consider a complex topology where the division of the 'father' contour is carried out with the skeleton principle using the circumscribed circles' definition.

\section{Simple topology change}

The principal idea is based on the detection of the nearest points between 'children' contours. Let $C_{k}^{1}$ the 'father' contour, and $C_{k+1}^{2,1}$ and $C_{k+1}^{2,2}$ the 'children' contours. We seek the points $q_{a} \in C_{k+1}^{2,1}$ and $q_{b} \in C_{k+1}^{2,2}$ such that the distance between these two points is minimal (Figure 7). Point $C$ is taken like a median of the line between $q_{a}$ and $q_{b}$ (Figure 7.b) and ray $R$ depends on the obtained minimal distance. Choosing $R=k \cdot \operatorname{dist}\left(q_{a}, q_{b}\right)$ where $k$ can vary between 1.5 and 2 following the various cases of topology and of the images resolution, we select a region of interest $\Omega$ in the 'father' contour, which represents the surface of the circle. In this region, we seek the nearest points to the 'father' contour $C_{k}^{1}$ where the exact place of the topology change is considered. If there are several sets of points with a similar distance, the set of the points that is closest in the centre $C$ of circle is considered. Then we divide the 'father' contour into two, basing on the founded points $p_{a}$ and $p_{b}$ (Figure 7.c), which describe the minimal distance

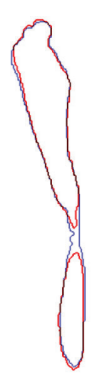

$a$

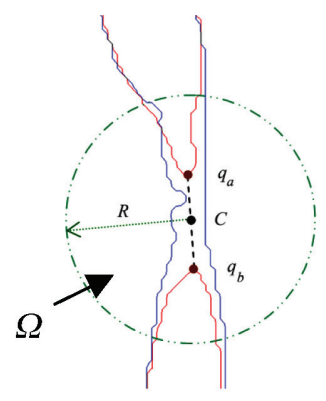

b

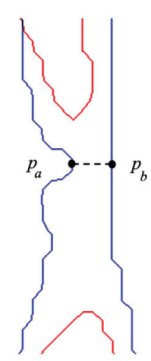

$c$
Figure 7 (a) 'Father' contour $C_{k}^{1}$ (blue), 'children' contours $C_{k+1}^{2,1}$ and $C_{k+1}^{2,2}$ (red); (b) Selection of the region of interest $\Omega$ according to the points $q_{a}$ and $q_{b}$, the closest between $C_{k+1}^{2,1}$ and $C_{k+1}^{2,2}$; and (c) Selection of division points $p_{a}$ and $p_{b}$ of contour $C_{k}^{1}$ based on minimal distance. 


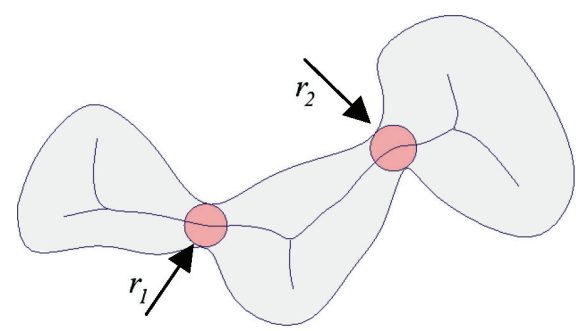

Figure 8 Detection of the points belonging to the circumscribed circles of the median axis having a minimal ray value.

in the region of interest. The line passing by the two points $p_{a}$ and $p_{b}$ permits to consider that the 'father' contour is divided into two, enabling to carry out the correspondence with the 'children' contours.

\section{Complex topology change}

If the 'children' contours are more than two, we cannot use the criterion of distance between them. The reasons are topological; it may be that separation occurs with successive contours, or with more complex contours. It is then preferable to seek to locate the separation regions directly in the 'father' contour. For this reason, we are based on the principle of the median axis of contour (Figure 8). In the $2 \mathrm{D}$ case, the median axis of a contour represents a whole of points that are inside or also outside the contour. These points are the centres of the circumscribed circles inside the region limited by the contour. From an 'anatomical' point of view, the calculation of the median axis is defining the 'skeleton' of the contour surface. Figure 8 shows, that the regions $r_{1}$ and $r_{2}$ represent the minimal surfaces that indicate the places of contour division. The problem of detecting the separation regions consists to find the circles of the median axis having a minimal value of ray. There are certain limits to this method. First of all, circles should not be considered having points belonging to contours that are relatively close, with the contrary case we are likely to locate toward local minima. In this case, the resulting topology will be erroneous. Nevertheless, there are cases in which separation occurs in places where they can be regarded as local minima. To solve this problem, it is essential to carry out a setting parameter of adequate selection of the circles according to the neighbourhood of the contour points. The experiment showed that this setting parameter is not necessary for many other cases.

The separation process of the 'father' contour is as follows: For $M$ 'children' contours, we need to define $M-1$ circles of the median axis of the 'father' contour. Initially we carry out the detection of the first circle having a minimal ray. Then we choose the points belonging to the circle. We make the division of contour as it is described in the preceding method. We continue in the same way until the moment when, for each generated contour corresponds only one 'child' contour. Finally, we carry out the adaptive triangulation of the parts of contours.

\section{Method validation}

In this section, we present the validation results of the reconstruction method in various cases. Figure 9 presents the algorithm that incorporates all the necessary operations to achieve a $3 \mathrm{D}$ reconstruction.

Procedure SurfaceGeneration (\& Container Cont)

$$
L_{k}^{n} \in \text { Cont }
$$

while $\underline{i}<n$

$$
\begin{aligned}
& \text { IndexingContours ( } \& \text { Layer } L_{k-1}, \& \text { Layer } L_{k} \text { ) } \\
& \text { if } i=1 \text { or } i=n \\
& \text { Close surface } \Omega \text { defined by all } C_{i} \text {, } \\
& \text { if } C_{i} \text { does not have surface } \Omega \text { generated by the } \\
& \text { indexing of contours. } \\
& \text { end if } \\
& \text { while } j<\text { correspondences number } \\
& \text { diff }=\text { difference of corresponding contours } \\
& \text { case selection diff } \\
& \text { case: } 0 \text { Adaptive } \\
& \text { Triangulation } \\
& \text { case: } 1 \text { SimpleDivision, AdaptiveTriangulation } \\
& \text { case: }>1 \text { ComlexeDivision, Adaptive } \\
& \text { Triangulation } \\
& \text { end case selection } \\
& \text { end while }
\end{aligned}
$$$$
\text { end while }
$$

The reconstruction method that we have presented is based on topological criteria, and it aims at answering the problem of the $3 \mathrm{D}$ reconstruction of the complex shapes present in the 2D images starting from 2D contours.

Figures 10 and 11 present the surface generation of a set of several consecutive contours with different topological cases. In the case of a rather complex topology, as the case of the teeth, we noted that there can be problems of correspondence between contours.

In Figure 12, we show the 3D reconstruction of a dental plaster part, very complex in terms of topology (molar teeth). In both cases, our algorithm identifies the regions of division and proceeds to an adaptive triangulation. We present in Figure 13 the surface generation of a dental plaster starting from $2 \mathrm{D}$ contours.

This method allows obtaining the $3 \mathrm{D}$ reconstruction of a more consistent and accurate model in comparison with the Marching Cubes algorithm, but it requires our intervention to solve certain difficult topological cases of contours correspondences.

\section{SPECIFIC 3D TREATMENTS}

The specificity of the obtained dental X-ray computed tomography images and the requirements of the specialists in this domain justify our motivation to develop specific treatments, allowing a reduced calculation time and efficient diagnosis. From the obtained 3D models (Maxillary and Mandible) by the Adaptive triangulation method, we present in this section, first, the automatic correction of 


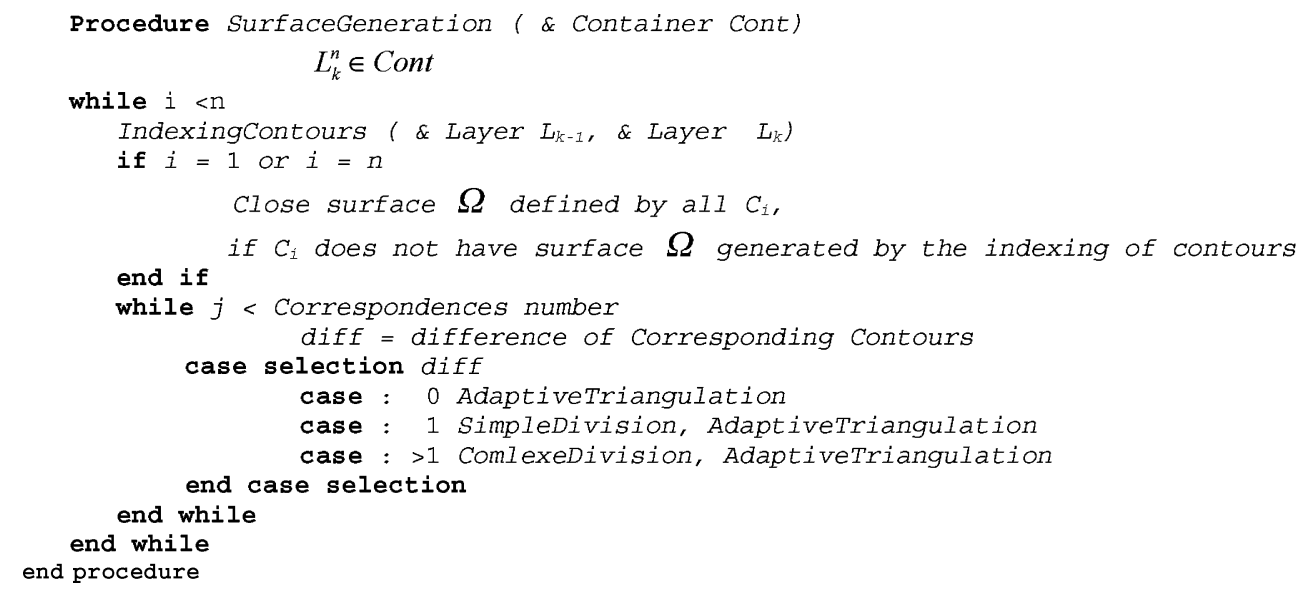

Figure 9 3D reconstruction algorithm from 2D contours.

the occlusion error and, second, a method that treats individually each tooth. This treatment is needed by the orthodontists to perform a medical examination.

\section{Automatic correction method of the occlusion error}

In our treatment method, we suppose that a dental plaster is composed of two parts: the Maxillary and the Mandible, as illustrated in Figure 1(a). To respect the natural positioning of the Maxillary and the Mandible, we have conceived a special box permitting to control the horizontality and the verticality of the two parts in relation to the scanner bed. Moreover, this box allows moving away the two parts according to the horizontal axis with the use of drawers. This last operation allows to get two separate sequences of 2D images. We can also measure the distance that separates the two parts with an unscrewed needles system.

This measurement can be obtained directly from the images according to the horizontal axis. The setting in occlusion is then given while displacing one of the two models in relation to the measured occlusal distance. As we were not able to scan the models in occlusion, we have been obliged to analyse the two parts with a distance between them, to avoid images that contain both the Maxillary and Mandible objects. In such a case, it would be very difficult to separate the models in an automatic way. Then, we measured the displacement distance with the modifiable reference marks. To put the models in occlusion in the ideal case without errors, we combine them under only one global 3D model. Then, we move the second model in relation to the $Z$ axis while using the occlusal distance, calculated from the images where the reference marks are located. Indeed, if $I_{m}$ is the number of the image with the first reference mark, $I_{n}$ the number of the image with the second reference mark and $R Z$ the distance in millimetres between two consecutive images, the occlusal distance dist $_{Z}$ is given by:

$$
\operatorname{dist}_{Z}=\left(I_{m}-I_{n}\right) \times R Z .
$$

We apply this translation for all points of the triangles of the second model. Every point $P(x, y, z)$ of the model is replaced by $P^{\prime}\left(x^{\prime}, y^{\prime}, z^{\prime}\right)$ where:

$$
x^{\prime}=x, \quad y^{\prime}=y, \quad z^{\prime}=z-\operatorname{dist}_{Z} .
$$

Some conditions can affect the horizontal and vertical positioning of the dental box during the analysis. A bad regulation of the scanner bed results a vertical slope (Figure 14.a). If the box is not aligned correctly in relation to the scanner bed, it will be analysed with a horizontal slope. These errors produce a vector $\mathbf{V}^{\prime}\left(x^{\prime}, y^{\prime}, z^{\prime}\right)$ of analysis instead of a vector $\hat{\mathbf{V}}(0,0,1)$. This vector is calculated with the two reference marks on the images $I_{m}$ and $I_{n}$. In an ideal situation, these points of reference marks should be confounded. While calculating the deviation $e_{x}$ and $e_{y}$ ac-

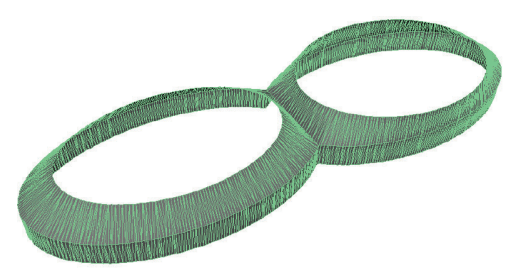

a

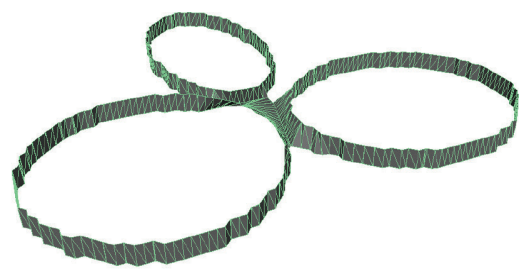

b

Figure $103 \mathrm{D}$ reconstruction of synthetic contours (a) 3D reconstruction of a synthetic model (topology with simple division) and (b) 3D reconstruction of a synthetic model (topology with complex division). 


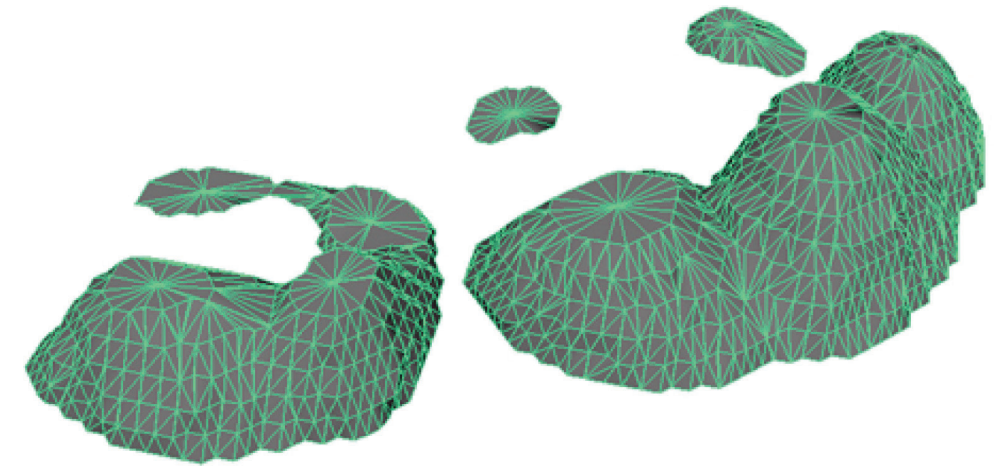

Figure 11 3D reconstruction of a set of six layers of 2D contours.

cording to the $X$ and $Y$ axis and the distance dist $t_{z}$ between the images $I_{m}$ and $I_{n}$ according to the $Z$, we model this error by:

$$
\mathbf{V}_{\mathrm{err}}=\mathbf{V}^{\prime}=\left[\begin{array}{lll}
e_{x} & e_{y} & \text { dist }_{z}
\end{array}\right]
$$

In these modelling conditions, we find the transformation matrix to correct the error represented by the vector $\mathbf{V}_{\text {err }}$ where $P=\mathrm{T} P_{\text {err }} ; P_{\text {err }}$ corresponds to the $P$ point of the transformed model by error vector $\mathbf{V}_{\text {err }}$; and $\mathbf{T}=\left[\begin{array}{ll}\mathbf{R} & \mathbf{t} \\ \mathbf{0} & 1\end{array}\right]$ is the transformation matrix. For the Maxillary model, we can represent the transformation matrix as:

$$
\begin{aligned}
\mathbf{T}_{\max i l}=\left[\begin{array}{cc}
\mathbf{R} & \mathbf{t}_{\max i l} \\
\mathbf{0} & 1
\end{array}\right] & =\left[\begin{array}{cccc}
r_{11} & r_{12} & r_{13} & t_{x(\max i l)} \\
r_{21} & r_{22} & r_{23} & t_{y(\max i l)} \\
r_{31} & r_{32} & r_{33} & t_{z(\max i l)} \\
0 & 0 & 0 & 1
\end{array}\right] \\
& =\left[\begin{array}{cccc}
r_{11} & r_{12} & r_{13} & 0 \\
r_{21} & r_{22} & r_{23} & 0 \\
r_{31} & r_{32} & r_{33} & 0 \\
0 & 0 & 0 & 1
\end{array}\right] . \quad(7)
\end{aligned}
$$

For the Mandible model, we have to put it in contact with the maxillary model. We perform this step using the

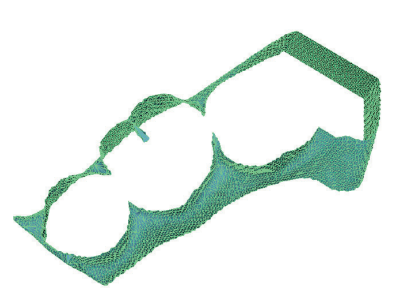

$a$

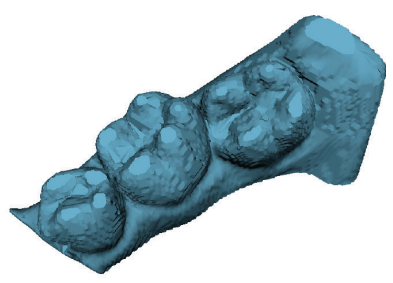

$b$
Figure $123 \mathrm{D}$ reconstruction of a teeth part starting from 2D contours. obtained occlusal distance:

$$
\begin{aligned}
\mathbf{T}_{\text {mand }}=\left[\begin{array}{cc}
\mathbf{R} & \mathbf{t}_{\text {mand }} \\
\mathbf{0} & 1
\end{array}\right] & =\left[\begin{array}{cccc}
r_{11} & r_{12} & r_{13} & t_{x(\text { mand })} \\
r_{21} & r_{22} & r_{23} & t_{y(\text { mand })} \\
r_{31} & r_{32} & r_{33} & t_{z(\text { mand })} \\
0 & 0 & 0 & 1
\end{array}\right] \\
& =\left[\begin{array}{cccc}
r_{11} & r_{12} & r_{13} & 0 \\
r_{21} & r_{22} & r_{23} & 0 \\
r_{31} & r_{32} & r_{33} & \| \\
0 & 0 & 0 & V_{\text {err }} \|
\end{array}\right],
\end{aligned}
$$

where $\left\|V_{e r r}\right\|=\sqrt{e_{x}^{2}+e_{y}^{2}+\operatorname{dist}_{z}^{2}}$ and

$$
R=\left[\begin{array}{l}
\hat{\mathbf{X}} \\
\hat{\mathbf{Y}} \\
\hat{\mathbf{Z}}
\end{array}\right]=\left[\begin{array}{lll}
r_{11} & r_{12} & r_{13} \\
r_{21} & r_{22} & r_{23} \\
r_{31} & r_{32} & r_{33}
\end{array}\right] \text {. }
$$

Finally, we calculate the common rotation matrix that allows the setting in occlusion of virtual models (Figure 14.b). The vectors $X, Y$ and $Z$ represent the lines of the rotation matrix.

We want to construct the three lines of the rotation matrix. Every line corresponds to an axis of the erroneous coordinated system. However, we compute the three axes of rotation. The first axis is known, because it corresponds to the error vector $\mathbf{V}_{\text {err. }}$. Thus, after normalization of $\bar{V}_{\text {err }}$, we have $\bar{Z}=\hat{\boldsymbol{V}}_{\text {err }}$ and,

$$
r_{31}=\hat{Z}_{x}, \quad r_{32}=\hat{Z}_{y}, \quad r_{33}=\hat{Z}_{z} .
$$

The second axis $\overline{\boldsymbol{Y}}$ is constructed from the global axis $\overline{\boldsymbol{Y}}_{w}=\left[\begin{array}{lll}0 & 1 & 0\end{array}\right]$; we have, then, $\overline{\boldsymbol{Y}}=\overline{\boldsymbol{Y}}_{\boldsymbol{w}}-\overline{\boldsymbol{Y}}_{\boldsymbol{w}} \cdot \overline{\boldsymbol{Z}} * \overline{\boldsymbol{Z}}$.

If we consider $d=\overline{\boldsymbol{Y}}_{\boldsymbol{w}} \cdot \overline{\mathrm{Z}}=Y_{w X} Z_{x}+Y_{w Y} Z_{y}+$ $Y_{w Z} Z_{z}$, we have $Y_{x}=Y_{w X}-d Z_{x}, Y_{y}=Y_{w Y}-d Z_{y}$, $Y_{z}=Y_{w Z}-d Z_{z}$, and after normalization of $\overline{\boldsymbol{Y}}$, we obtain:

$$
r_{21}=\hat{Y}_{x}, r_{22}=\hat{Y}_{y}, \quad r_{23}=\hat{Y}_{z} .
$$

We compute the last axis with the cross product of the two calculated axes: $\bar{X}=\bar{Z} \times \bar{Y}$. We normalize the vector $\bar{X}$, and we obtain the first line of the rotation matrix $\mathbf{R}$ :

$$
r_{11}=\hat{X}_{x}, \quad r_{12}=\hat{X}_{y}, \quad r_{13}=\hat{X}_{z} .
$$




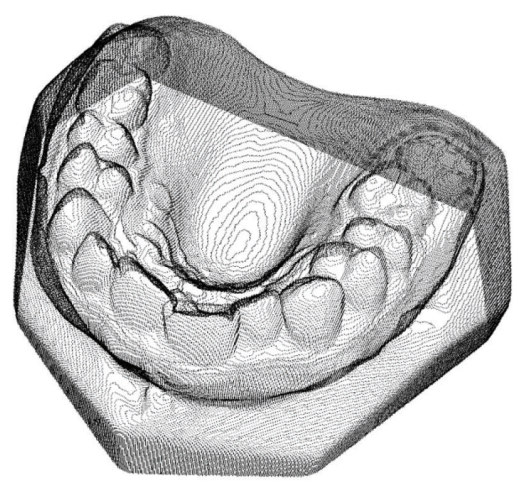

$a$

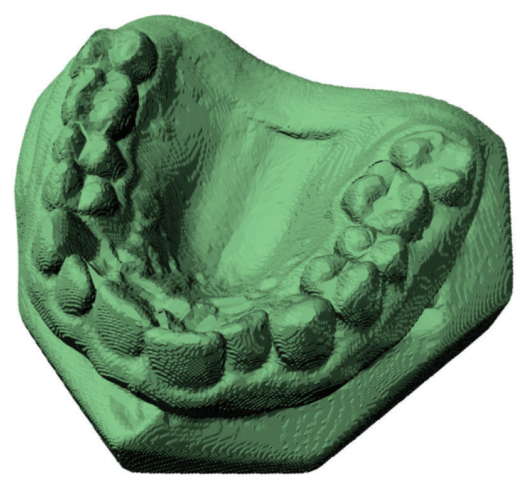

b

Figure 13 Creation of a 3D dental plaster starting from 2D contours: (a) sets of contours and (b) triangulation of contours and generation of the grid surface.

So we have computed the rotation matrix $\mathbf{R}$. To get the corrected model, it is necessary to apply the corresponding transformation matrixes ( $\mathbf{T}_{\text {maxil }}$ and $\left.\mathbf{T}_{\text {mand }}\right)$ on every point of the sub-models. This method allows an automatic occlusion correction on the virtual model that avoids the orthodontists to do a second or third scanner for the same patient.

\section{Segmentation and individual treatment of teeth}

In the dental field, a 3D teeth model — in which each tooth can be manipulated individually-is an essential component for the simulation on orthodontic surgery and treatment. To reconstruct such a $3 \mathrm{D}$ teeth model from $2 \mathrm{D}$ images, we need to localize and define the accurate region of each tooth from images' sequence.

The complexity of the teeth makes the task difficult to elaborate an automatic method for the treatment of the teeth separation. In Heo et al. (2004), a segmentation method based on b-spline curve of tooth is presented on a $2 \mathrm{D}$ image. The authors discuss the obtained results on 2D image, but the difficulty for $3 \mathrm{D}$ reconstruction of the teeth separation is not presented. The algorithm presented by Kondo et al. (2004) is based on the dental arch obtained from $2 \mathrm{D}$ images. This arch is used as reference to compute the dental model. But the evolution on the sequence can induce errors, and the 3D model of teeth can be deformed.
The used method of segmentation (Djemal et al. 2002) for the rebuilding of the Mandible and the Maxillary gives good results on the totality of the model but does not allow the intervention of the orthodontist to extract and rebuild individual teeth.

For the orthodontists' needs concerning an individual tooth treatment, we have developed a teeth localization method. It is necessary to mention that this dental treatment is a procedure where the orthodontist cuts the teeth of a plaster model manually, to reposition them and to extract important information for his patient's treatment. Our method is based on the use of a numbered grid with the numbers of the teeth. This grid is introduced on the plaster images' sequence.

Initially, one manually adjusts the grid for the first and the second image of the sequence to predict a grid that will be used automatically for the rest of the images' sequence. The advantage of this method is that it makes it possible to locate and extract only one tooth from the sequence by choosing its corresponding grid area. If one wishes to rebuild the entire $3 \mathrm{D}$ model, it is necessary to repeat the same procedure for each tooth.

The horizontal position of plasters in relation to the $Z$ axis, according to which the scanner bed moves, allows the regions of the contacts of teeth to be practically at the same place on the images' sequence. It is then possible to define the regions where each tooth is located while displacing

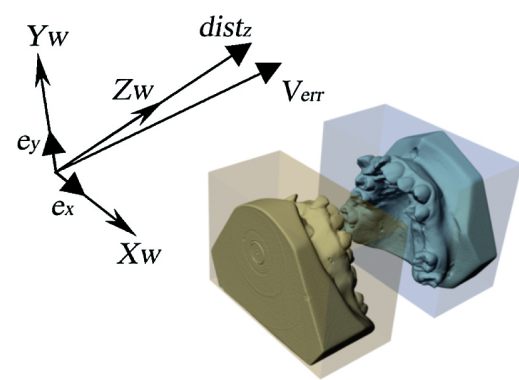

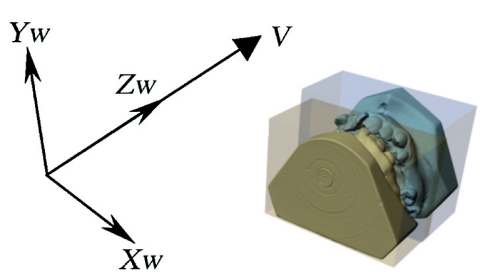

$b$

Figure 14 (a) Rotation of the model in relation to the error vector and (b) automatic translation of the Mandible. 


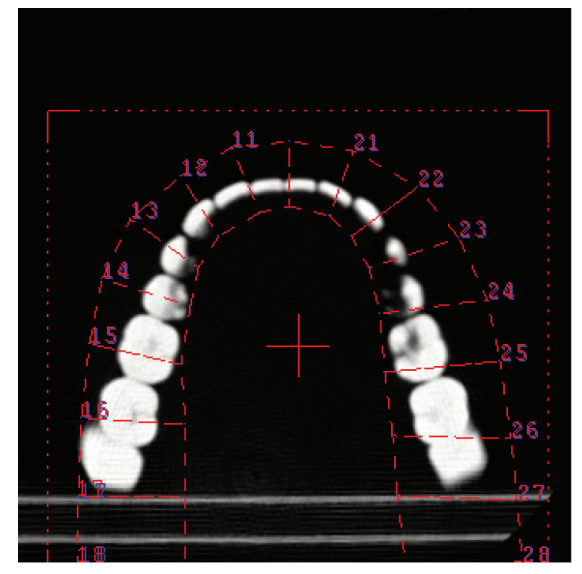

a

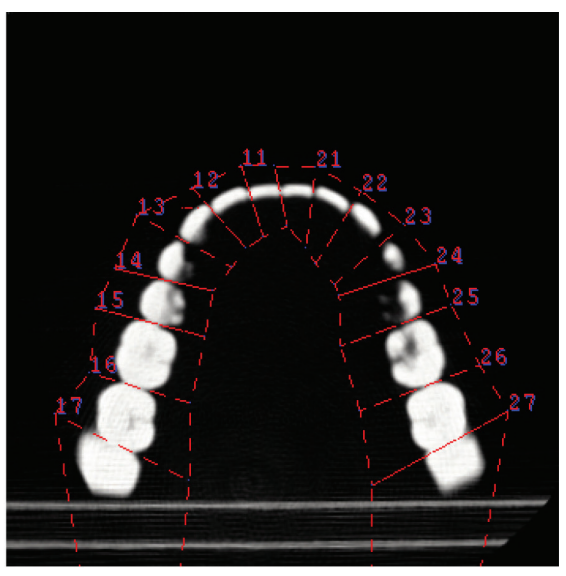

b

Figure 15 Numbered grid used for localization and segmentation: (a) before the calibration and (b) after calibration.

the characteristic points that define the numbered regions of the grid. For the rare cases where the teeth leave the specified region (slope of a tooth in relation to the scanner bed) during the images evolution, we have developed a system that records key grids. Then, we can define a new grid, inherited from the previous and displace the concerned points to include again a tooth in its region (Figure 15). For each tooth, we apply a mask that segments its region. The final step is the $3 \mathrm{D}$ generation of each tooth and the combination of all 3D models of teeth under only one model (Figure 20). The experience showed that, in $90 \%$ of the cases, only one grid is sufficient for the dental segmentation. The positioning of the segmented teeth is automatic because the $3 \mathrm{D}$ objects are created taking into account the topology of the images' sequence.

\section{DIVITOR APPLICATION}

The DIVITOR application allows at the first time the dental plaster receipt, the digitization, the data organization in a database and the transfer of the examinations toward the doctors' laboratories. In the second time, it provides the tools for the specific treatments and telediagnosis of the patient's examinations.

\section{Client/Server protocol}

The consequent number of dental plasters in orthodontics raises a reflection concerning the best methods of digitization. Indeed, a simple demand requires the digitization of $2,000-3,000$ plasters per month; this is just to satisfy about $40-50$ orthodontists. After the scan of dental plasters, a DICOM server is used to receive the images' sequence.

When the plasters are received by the orthodontists, additional information is inserted into the database. The query is achieved by using the XML protocol (McHugh and Widom 1999). Information concerning patients is extracted from DICOM images, then it is integrated in a structured database. This base is structured in a manner to make it possible to easily obtain reliable answers by using the XML protocol.

The model resolution is $0.256 \mathrm{~mm}$. Some previous research demonstrated that this resolution is clinically acceptable for the treatment of this type of examinations (Santoro et al. 2003; Quimby et al. 2004). A communication

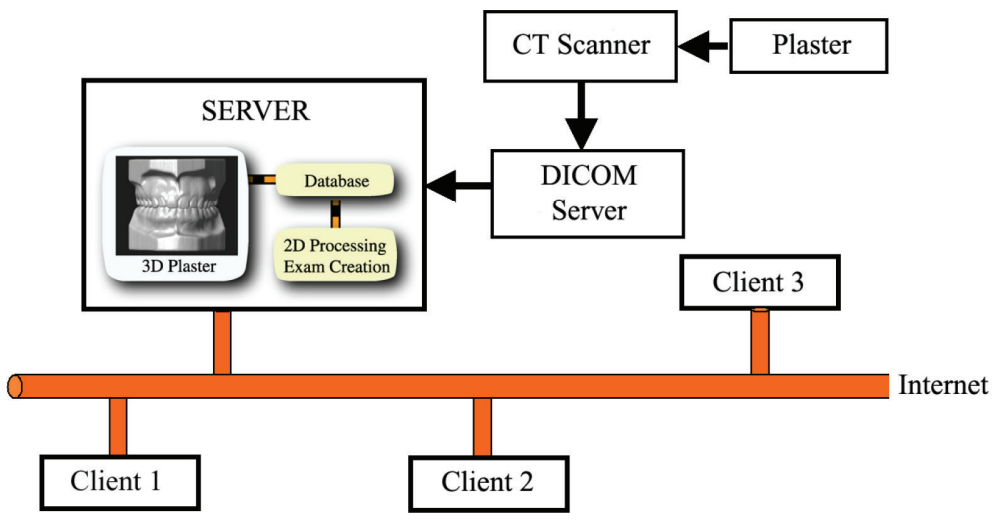

Figure 16 General diagram of DIVITOR. 

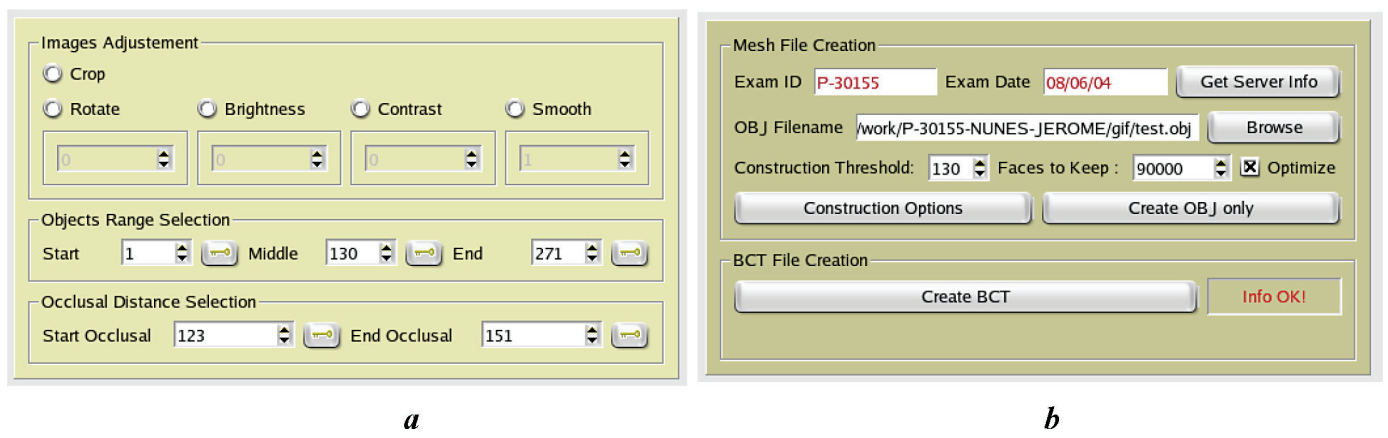

Figure 17 (a) Image processing, image keys settings and (b) database communication and examination creation.

system via web services between the server application and the database allows the addition of supplementary information in the examinations (Figure 16). This information is added during the receipt of plasters, and it is necessary for the logistical management. Then the examinations are stored into the database, remaining at the disposal of orthodontists.

\section{DIVITOR Server}

The digitization of the dental plasters as well as the creation and availability of the examinations make the major part managed by the DIVITOR Server. We have defined a scan protocol to allow the quality and the resolution of the $3 \mathrm{D}$ models generated from DICOM images. This protocol is recorded at the CT scanner console. Generally, in radiology services, the standard image format DICOM (EMA 1993) is used with the CT scanners. A high pixel resolution and a special header where we can save additional information characterize this file format. We noted that the different constructors of CT scanners use their own configurations for the exploitation of DICOM images.

The existence of different formats of DICOMS images as DICOM2 and DICOM3 led us to adapt an algorithm to allow compatibility between images produced by different CT scanners and our 3D reconstruction system. This algorithm takes into account the necessary information of DICOM headers and saves them to have a generic header compatible with our system. In this header, other infor- mation related to the management of the examinations is added. The DICOM images are then converted to flexible and light format, allowing also automatic consultation via a web server.

As is mentioned above, one advantage of this application is the speed of creation of the dental examinations. To improve that, we also created a 3D render system. Firstly, we insert the necessary parameters needed for the creation of the 3D examination, and secondly, we save them under a special file format (batch file). In such a way, we can save several examinations very quickly without executing the procedure of the $3 \mathrm{D}$ creation. Finally, we can start the execution of the creation of several examinations. This render concept is used extensively on applications of computer graphics and allows a better organization of data and also decreases the calculation time of 3D dental examinations. Consequently, the generation of $3 \mathrm{D}$ models takes place massively in one chosen moment (e.g. the night).

\section{DIVITOR Client}

The internet connection offers to orthodontists several advantages. Indeed, they can access to the database via the server every time they want to recover the examinations and save locally the modifications concerning data patients.

The DIVITOR client allows orthodontists to realize many treatments from their laboratories as data transfer, data visualization, patient diagnosis and specific dental examination treatments. Indeed orthodontists have the

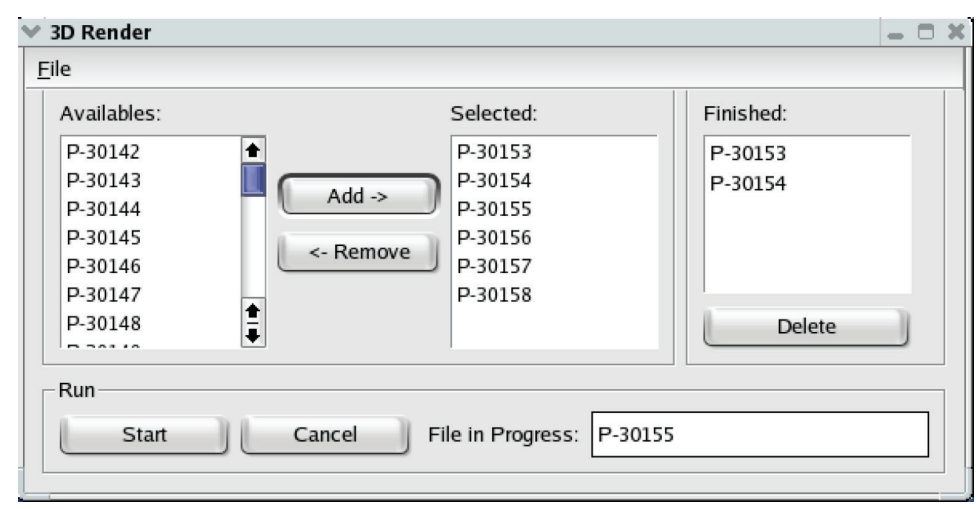

Figure 18 3D Render management and execution. 


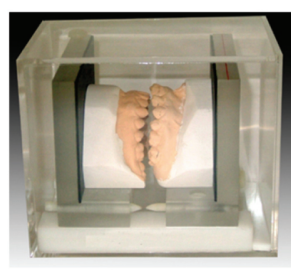

a

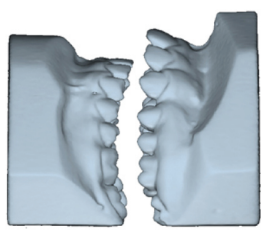

d

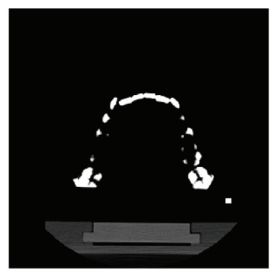

b

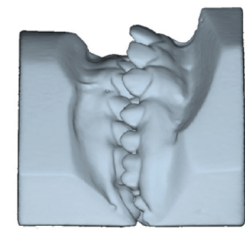

e

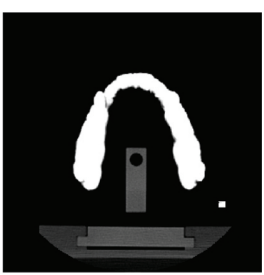

c

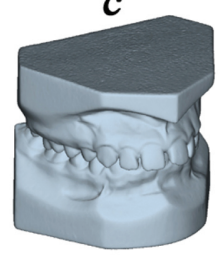

f
Figure 19 (a) The dental box of the model orientation, (b and c) the characteristic images with the unscrewed reference marks, (d) 3D model of plaster and (e and f) setting in occlusion.

possibility to download dental examinations with secure communication from the server database. Then they can do an automatic research of the patient's examination of which they want to make the diagnosis. The client also gets a secured access to the fields of the examination to add or fill some additional information.

The specific treatments can be directly achieved on the $3 \mathrm{D}$ model like the automatic correction of the occlusion error and the individual treatment of teeth. Visualization and display of the 3D models are performed with a simple and efficient way, allowing the space orientation of the image. So, with the help of corresponding buttons, rotation, pan and zoom of the camera help the orthodontists to visualize easily the dental examinations. The orthodontists can do rotations and translations of the Maxillary or Mandible model to have a complete image of the occlusion. In addition, this application gives the possibility to do virtual cuts of the 3D model. These cuts allow visualizing easily the points of contact of the teeth in a specified region (oc- clusion) while removing all surfaces that exist before this region.

It is also possible to do some different types of measures: mesiodistal, vestibulolingual, height, inter-canine and inter-molar. Administrative procedures concerning the patients often require the justifying of the dental examinations, by providing simple photos. Disposing a print/save functionality, they can print or save different views of the model without using a camera as it used before.

\section{EXPERIMENTAL RESULTS VIA DIVITOR APPLICATION}

In this section, we illustrate our work by results obtained on real X-ray computed tomography images. The procedure of a dental examination creation is very convenient and quiet fast. Indeed, the main server application enters in communication via an Internet connection with the server's database. Then we interrogate the database by providing the code and the date of the examination. In this way, we collect all additional information on the examinations, which is saved in the database during the receipt of plaster. As soon as the communication is finished and after possible image processing (Figure 17.a) like modify brightness and contrast, apply smooth filter and perform a rotation on the set of images, we can pass to the examination creation as illustrated in Figure 17.b.

Finally, the server offers a 3D render management function (Figure 18), with the possibility to save and execute one or several files of $3 \mathrm{D}$ render type.

We generate first the Maxillary model and secondly the Mandible model. To get the final 3D model, we combine the two models using the corrected occlusal distance. Figures 19.a, 19.b and 19.c show the steps of digitalization and dental plaster acquirement. We can note (Figure 19.d) the satisfactory result of the reconstruction of the digital 3D plaster models. The result of the setting in occlusion after a possible correction is shown in Figures 19.e and 19.f.

The segmentation result of the individual treatment of teeth is shown in Figure 20. This result is obtained on the

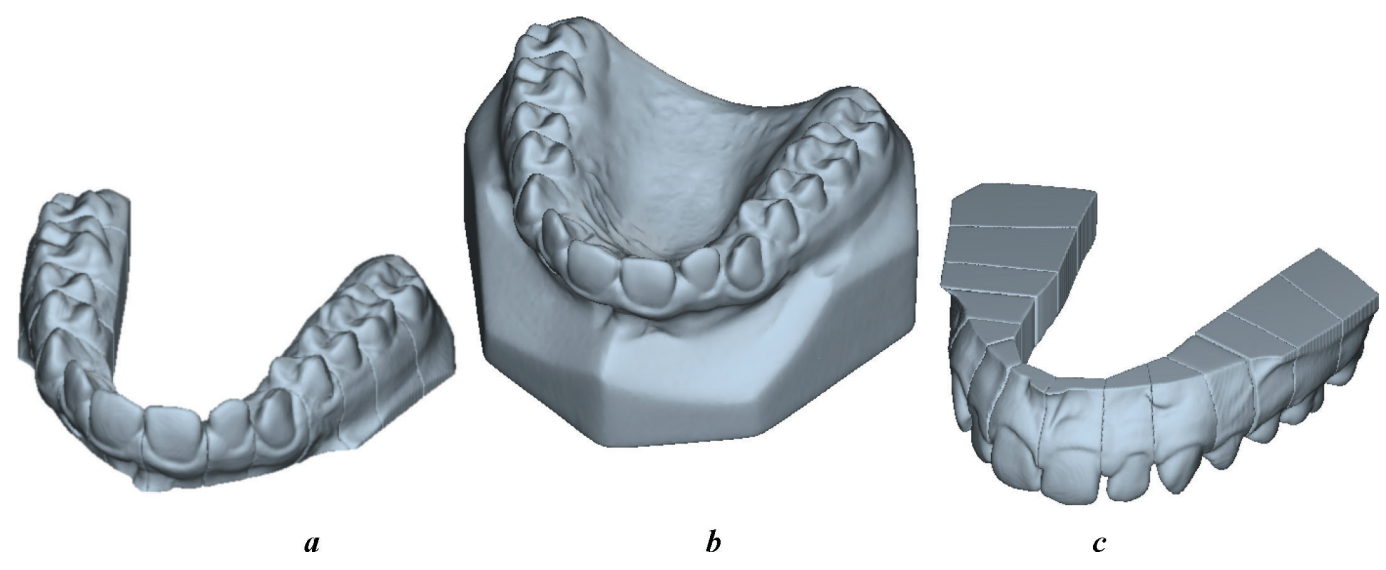

Figure 20 Segmentation of the teeth: (a and c) two views of the teeth of the segmented maxillary and (b) maxillary part before the segmentation. 

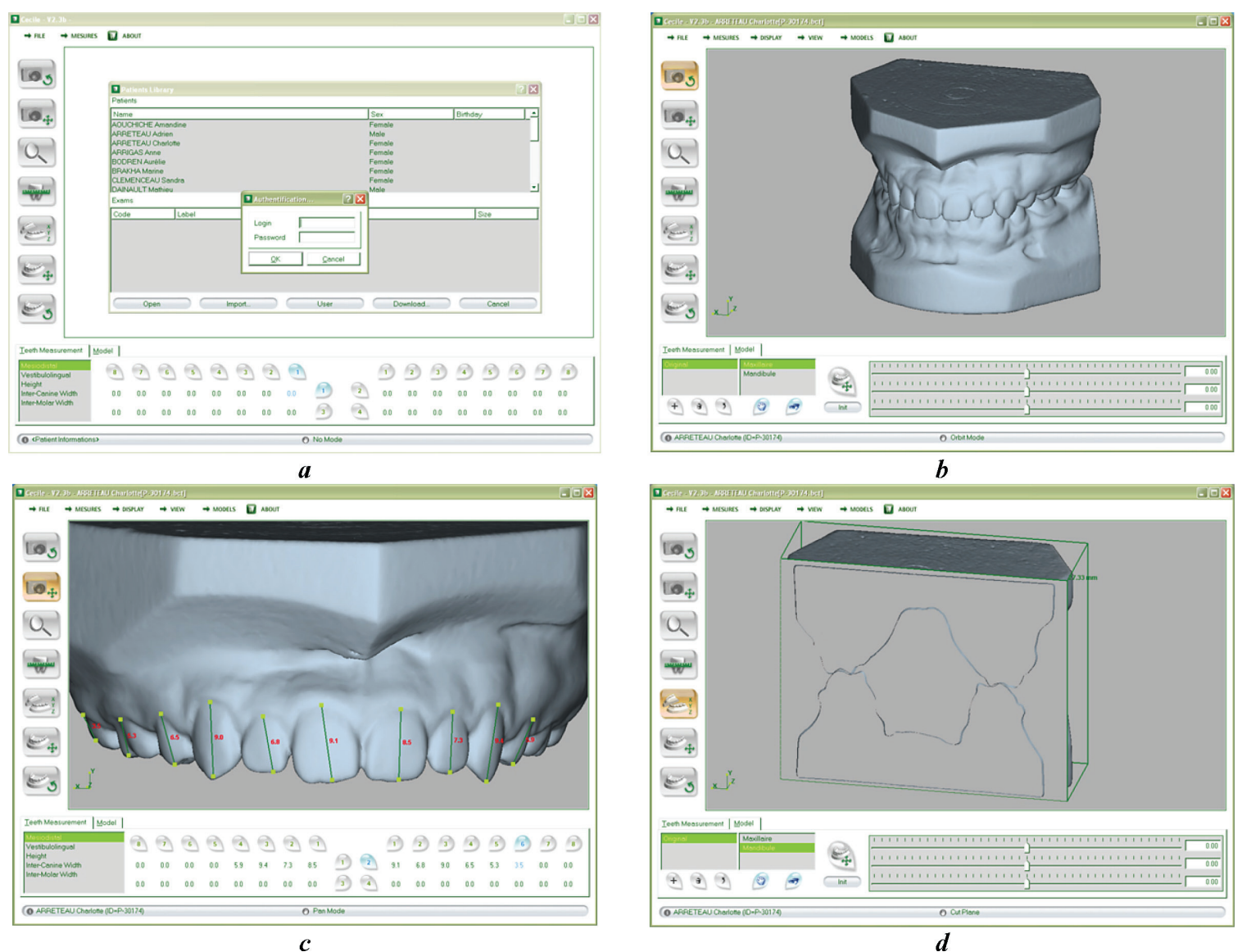

Figure 21 Client operations: (a) examinations download and management, (b) model transformation, (c) taking of measures and (d) virtual cut of the $3 \mathrm{D}$ model.

Maxillary (Figure 20.b). Figures 20.a and 20.c show two views of the teeth of the segmented maxillary.

Orthodontists, as we explained in the previous sections, can achieve several operations either in their laboratories or via Internet. Indeed, the orthodontist benefits from a confidential access (Figure 21.a) to the database managed by the server.

The orthodontist can make transformations of the 3D model on the sub-parts (Maxillary, Mandible) and also do rotation, translation and zoom of the 3D model (Figure 21.b). An interface is developed to facilitate the teeth measurements as illustrated in Figure 21.c. These operations concern mesiodistal, vestibulolingual, height, inter-canine and inter-molar measures. Figure 21.d presents a virtual cut of the 3D model. By selecting the face of interest, we can progress dynamically the virtual cut to arrive to the region of the model of which we want to study the occlusion.

\section{CONCLUSION}

In this article, we have presented a 3D specific dental plaster treatment system for orthodontics domain. In this aim, we proposed an adaptive triangulation method for the 3D reconstruction of structures with complex topology, start- ing from a sequence of real X-ray computed tomography images. This method is applied to the $3 \mathrm{D}$ reconstruction of a dental plaster that allows obtaining a consistent $3 \mathrm{D}$ model and accurate reconstruction. From an obtained 3D model, we have presented specific treatments allowing the automatic correction of the occlusion error and the individual treatment of teeth needs by the orthodontists.

The performance of the proposed method of $3 \mathrm{D}$ reconstruction based on adaptive triangulation and specific treatments depends on the accuracy of obtained contours on X-ray computed tomography images.

The obtained results are presented and discussed via the DIVITOR client/server application. This application allows the digitization and the visualization, and provides the tools for the specific treatment and telediagnosis of the patient's examinations.

\section{ACKNOWLEDGEMENTS}

We thank Cyril Cosensa and Dr Alain Decker for their scientific help and the validation of the dental examinations. We also thank Mr Charlie Elkouby and the members of the Bibliocast society for their advices and their vital contribution to this work. 


\section{REFERENCES}

Boissonnat JD, Geiger B. 1992. Three dimensional reconstruction of complex shapes based on the delaunay triangulation. Research Report No. 1697, INRIA, Robotic, Image and Vision, France.

Djemal K, Bouchara F, Rossetto B. 2002. Image modeling and region-based active contours segmentation.In International Conference on Vision Modeling and Visualization VMV 2002, 363-370, Erlangen, Germany.

Djemal K, Paris S, Grimaldi M, Puech W, Rossetto B. 2003. Reseau d'Imagerie Medicale et d'aide au diagnostic (R.I.M.A.D), In International Conference SETIT, SOUSSE, TUNISIA.

Djemal K, Puech W, Rossetto B. 2006. Automatic active contours propagation in a sequence of medical images. Int $\mathcal{F}$ Image Graph, 6(2):267-292.

EMA 1993. Standard publication digital imaging and communication medicine, Dicom.

Fetita C, Preteux F. 2000. Bronchial tree modeling and 3D reconstruction. In SPIE Conference on Matheiago, CA.

Garland M, Heckbert P. 1997. Surface Simplification Using Quadric Error Metrics. In Proceedings of SIGGRAPH 97, ISBN:0-89791-896-7, 209-216.

Heo H, Chae OS. 2004. Segmentation of tooth in CT images for the $3 \mathrm{D}$ reconstruction of teeth. In Proceeding of SPIE, Image Processing: Algorithms and Systems III, 5298, 455466.

Klein R, Schilling AG, Straber W, 2000. Reconstruction and simplification of surfaces from contours.Graph Models, 62(6):429-430.

Kondo T, Ong SH, Foong KWC. 2004. Tooth segmentation of dental study models using range images, IEEE Transaction on Medical imaging, 23(3):350-362.

Lee C-Y, Antonsson EK. 1999. Surface reconstruction of etched contours. In Technical Proceedings of the International Conference on Modeling and Simulation of Microsystems, 117-119.

Lorensen WE, Cline HE. 1987. Marching cubes: A high resolution 3D surface construction algorithm. In Proceedings of the 14th Annual Conference on Computer Graphics and Interactive Techniques ,63-69, ACM Press.
McHugh J, Widom J. 1999. Query optimization for XML. In Proceedings of International Conference of Very Large Database, 315-326, Edinburgh, Scotland.

Malladi R, Sethian J, Vemuri B. 1995. Shape modeling with front propagation: a level set approach. IEEE Trans Pattern Anal Machin Intell, 17:158-175.

Marcel TJ. 2001. Three-dimensional on screen virtual casts. $A m \mathcal{F}$ Orthod Dentofacial Orthop, 119(6):666-668 .

Motohashi N, Kuroda T. 1999. A 3D computer aided design system applied to diagnosis and treatment planning in orthodontics and orthognatic surgery. Eur $\mathcal{F}$ Orthod, 21(3):263-274.

Oliva JM, Perrin M, Coquillart S. 1996. 3D reconstruction of complex polyhedral shapes from contours using a simplified generalized Voronoi diagram. Comput Graph Forum, 15(3):C397-C408

Persiano RM, Comba J, Barbalho V. 1993. An adaptive triangulation refinement scheme and construction. In Proceedings of the VI Brazilian Symposium on Computer Graphics and Image Processing (SIBGRAPI).

Quimby M, Vig K, Rashid RG, Firestone AR. 2004. The accuracy and reliability of measurements made on computer-based digital models. Angle Orthod, 74(3):298-303.

Redmond WR. 2001. Digital models: A diagnostic tool. 7 Clin Orthod, 34(6):386-387.

Rey D, Delingette H, Subsol G, Ayache N. 1999. Using continuum mechanics operators for detection and quantification of evolving processes in 3D medical images. EUROMECH'99, 185-188.

Santoro M, Galkin S, Teredesai M, Nicolay O, Cangialosi, TJ. 2003. Comparison of measurements made on digital and plaster models. Am $\mathcal{F}$ Orthod Dentofacial Orthop, 124(1):101-105.

Scheidegger CE, Fleishman S, Silva, CT. 2005. Triangulating point set surfaces with bounded error. In Symposium on Geometry Processing, 63-72.

Stämpfle M. 1999. Dynamical systems flow computation by adaptive triangulation methods. Computing and Visualisation in Science. Springer-Verlag: Berlin, 2:15-24 .

ter Haar BM. 1999. Scale-space theory for multiscale geometric image analysis. In IEEE Conference on Computer Vision Pattern Recognition. 

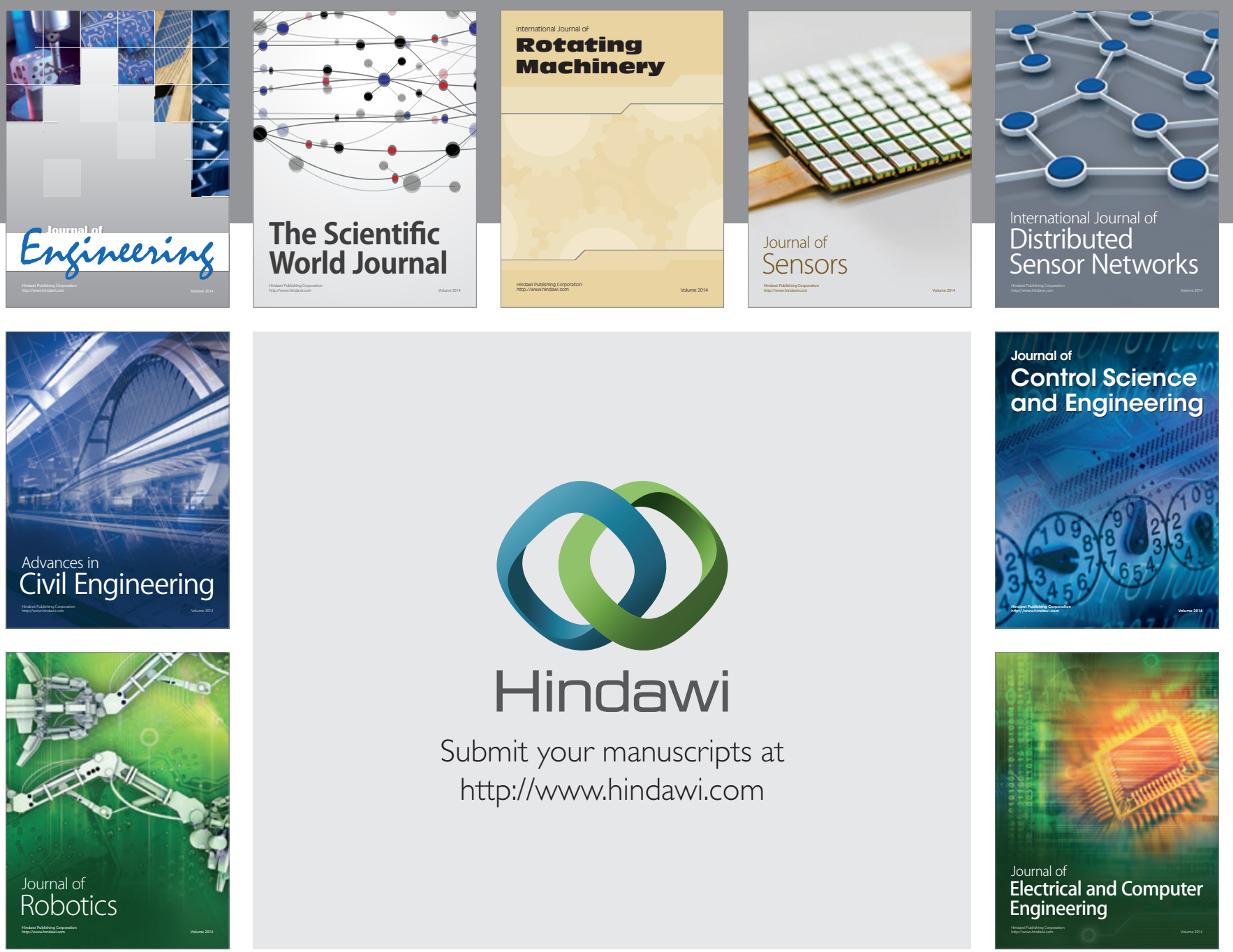

Submit your manuscripts at

http://www.hindawi.com
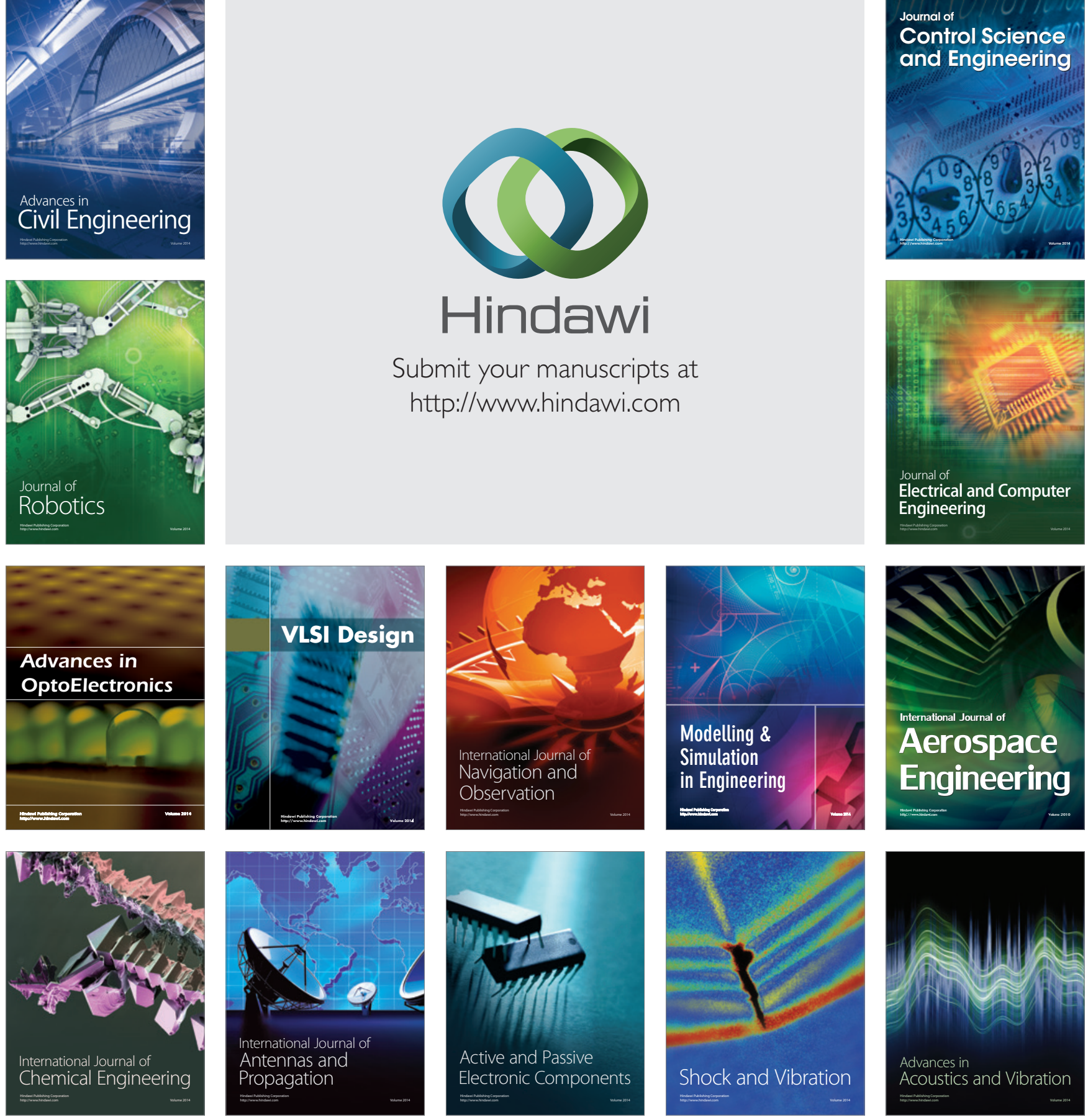